\title{
Application of Fun Virtual Test in Experimental Teaching*
}

\author{
Zhibang Yang ${ }^{1}$, Chen $\mathrm{Xu}^{2}{ }^{*}$, Yi Wang ${ }^{1}$, Xichuan Deng ${ }^{1}$, Jia Liu ${ }^{1}$ \\ ${ }^{1}$ Pathogen Biology and Immunology Laboratory, Experimental Teaching Center of Basic Medicine, Chongqing Medical University, \\ Chongqing 400016, China \\ ${ }^{2}$ Office of Academic Affairs, Chongqing Medical University, Chongqing 400016, China
}

\begin{abstract}
Objective Production of entertaining virtual test in Medical Microbiology and evaluation of its application in experimental teaching. Method: Considering the characteristics of medical microbiology experimental teaching, Photoshop was used to design the two network virtual experiments: "Observation of Bacterial morphology and dyeing by Gram staining" and "Isolation and identification of enteric pathogens". Obstacles were set according to the required techniques and error-prone operations. Students were scored based on the required experimental techniques. Backstage automatic scoring and feedback were set. Virtual experiment was used in the class, after-school review, and final assessment. Results: Students and teachers generally agreed that the virtual experiment can clearly demonstrate the whole experimental process, and it can help the operator remember experimental procedures with fun. Obstacles are mainly set with the middle-stage difficulties. There is no significant difference between experimental evaluation and practical examination results $(\mathrm{P}>0.05)$.Conclusion: Virtual test of medical microbiology was produced and applied in the experimental teaching with satisfactory results.
\end{abstract}

Index Terms - medical microbiology, virtual experiments, flash technology, internet, experimental teaching

\section{Introduction}

With the rapid development of information science and technology, application of computer, internet and virtual reality technology is advancing rapidly, leaving a huge impact on all aspects of society. In the field of medicine, virtual reality technology has been used in the diagnosis and prognosis of diseases [1-3]. In the field of higher education, computer, internet and virtual reality technology is becoming an effective instructional media and tool to give the teaching mode a profound reform [4]. In the experimental teaching, virtual experiment uses computers and simulation software to simulate the experimental environment and processes, so that students can have experiments on computers to preview and review procedures. Some large virtual experiment software can even replace some of the high-loss and high-risk real experiments.

Medical microbiology is a special discipline involving biosafety that has caused international laboratory concerns about biosafety since the 2003 SARS epidemic. A series of regulations were developed for the protection of individuals, including laboratory staffs, and environments. However, teaching for a larger amount of students would be difficult due to limited equipment and safety concerns. Therefore, building virtual medical microbiology experiment is valuable in application. In this study, internet virtual test of medical microbiology was produced using computer software to show the real experiment environment and simulate procedures and results. The application in experimental teaching of medical microbiology help students get easy access to proper training in and outside class, and relieve the pressure of school hardware.

\section{Materials and Methods}

\subsection{Virtual experimental design}

According to medical microbiology experiment outline, two virtual tests "observation of bacterial morphology and dyeing by Gram stain" and "isolation and identification of enteric pathogens" were designed. Experiment scenarios were set according to each experiment procedure. Obstacles were set up based on required techniques and error-prone procedures for high school students. Students were scored based on the importance of required techniques. The score and feedbacks were automatically recorded.

\subsection{Experimental scenario setup}

The experiment was on the bench, in Pathogen Biology and Immunology Laboratory, Experimental teaching Center of Basic Medicine. The experiment equipment was the operating scenario to fully experience the experiment.

\subsection{Experimental procedure setup}

In accordance with the teaching requirements, the main procedures are: 1) aseptic technique that operates nearby alcohol lamp flame or in biological safety cabinet, burns the inoculation loop (pin) and tube open before and after operation, and tube plugs can not be left on the bench; 2) the bacterial smear was produced from smear to drying, and then fixed in the "observation of Bacterial morphology and dyeing by Gram staining" experiment. Gram staining procedure was primary dyeing, mordant, bleaching, restained, microscope (oil immersion) observation and determination; 3) The operating procedure for "Isolation and identification of enteric pathogens" were intestinal fecal sampling, identification and inoculation by intestinal bacteria selection, suspicious colonies selection after culture, biochemical identification, serological identification, and isolates determination. The virtual

\footnotetext{
*Author: Zhibang Yang, male, 1948, Professor / Ph.D. Tutor, research directions: multimedia technology in microbiology teaching.

E-mail: Dryangfm365@ sina.com.cn.

Corresponding author: Chen Xu, male, April 1965, Professor / Ph.D.; research direction: medical education management.

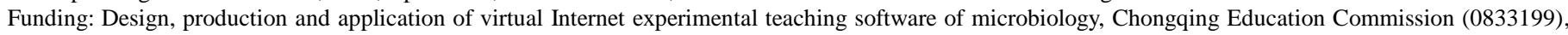
China
} 
experiment systemically showed the whole experiment procedures for students to experience.

\subsection{Obstacles setup and scoring}

In accordance with requirements of experimental teaching, students are required to learn laboratory aseptic techniques, so dressing and laboratory biosafety rules were set up as obstacles. The key steps and error-prone procedures of "observation of bacterial morphology and dyeing by Gram staining" were the griping of strain tube and vaccination ring, fixing method, dyeing order, and result determination. The key steps and error-prone procedures of "isolation and identification of enteric pathogens" were medium selection, suspicious colony selection, biochemical reaction determination, and isolates identification. Once there is error, scores would be deducted based on its importance. The experiment would automatically end if there were 3 mistakes. There are 100 points in total.

\subsection{Production of virtual internet software [1]}

Users operate in a manual manner. Internet software is consisted with subsystems of message processing, process control, scenarios, and backstage management. Message processing is the core part. It detects and responses to messages to be processed.

Process control subsystem is responsible for initializing the operating processes, including windows icons, width and height, window type, window title and window style. Scenario subsystem contains various characters, props, and scripts in certain order and manages stage control. Back stage management subsystem is responsible for data processing and information feedback. Photoshop sets up scenarios and Flash produces a cartoon-style animation. Action Script in Flash MX2004 provided appropriate programs and presented with ASP dynamic web page. An integrated database system was established to record the effects of applications, including trial size, operating results, as well as feedbacks.

\subsection{Application of virtual experimental internet software}

The software was installed in the experimental computers for students to operate. It was linked to the website of teaching center for students to preview and review after class and it was analyzed based on backstage feedback. Students were assessed and compared with experimental class scores and actual operation scores to analyze the differences using SPSS statistical software.

\section{Result}

\subsection{The application in experiment class}

In the first experiment class after "observation of bacterial morphology and dyeing by Gram staining", students used this software and they generally believe this virtual experiment can show the whole experiment process and can help the operator to remember all important points in the experiments with interest.

\subsection{After-school review of the experimental}

After upload the "Observation of Bacterial morphology and dyeing by Gram staining" on web page, 167 students had clicked, including 141 students $(84.5 \%)$ with completion, 16 students $(9.6 \%)$ who quitted because it had exceeded the maximum error number, and 10 students $(5.9 \%)$ who quitted without completion. There were 89 students $(63 \%)$ achieving a score of 100 points, 32 students $(23 \%)$ with a score between 80 and 100 points, 14 students $(10 \%)$ with a score between 60 and 80 points, and 6 students (4\%) with a score of less than 60 points. The difficulty coefficient of this software was 0.64 , which was a middle-level difficulty. Combined with performance in experiment class, it indicated that the virtual experiment software objectively showed how well students had learnt about the experiment. Evaluation for software could help students review the content and operation in experiment class while having fun. After school, students took a little bit time to review contents and the operation procedures of the experiments and knew how well they had learnt through score feedbacks. The drawbacks included the mechanical steps and fewer key procedures. Furthermore, the difficulty was just at medium level. The effect would be greatly improved if the background music selection was available.

\subsection{Application in exams.}

These two softwares were applied in experimental exams for 162 students in the department of Pediatrics and department of basic medicine. There were 92 students (56.7\%) achieving 100 points, 47 students $(29.0 \%)$ achieving scores between 80 and 100 points, 13 students $(8.0 \%)$ achieving scores between 60 and 80 points, and 10 students $(6.3 \%)$ achieving scores less than 60 points (Figure 4). The difficulty coefficients of dressing and laboratory biosafety regulation were 0.81 and 0.78 . The difficulty coefficients of Gram staining order, results determination, medium selection were between $0.6-0.7$. The difficulty coefficients of Gram staining fixing method, suspected colony selection, biochemical reaction determination, and isolates identification were between $0.5-0.6$. There was no significant difference $(\mathrm{P}>0.05)$ between practical examination and software test results, which was in consistent with the performance in class with distinguished grades. The drawback was the limited operation change. Students who took the examination later might get higher scores.

\subsection{Peer review.}

After actual operation, six senior teachers thought that the software screen is clear and the scenarios are real to show the whole process of experiment operation. Besides, the obstacles reflected the key steps of the experiment and error-prone procedures with animation, human-computer interaction and data management for review and preview. If the obstacles were increased, coupled with appropriate music and further improvement, it would be valuable for application.

\section{Discussion}

With the development of computer applications, multimedia and internet technology, research and development of education software has made great progress. The types of educational software are gradually increasing and applied. In 2005, the United States successfully applied education software in military and medical personnel training [6]. Many renowned 
universities have established online virtual labs, such as Virtual Physics Laboratory at Northwestern University, Physics Laboratory at University of Oregon, and Physics Teaching/Demonstration Laboratory at Taiwan Normal University. Online simulation starts in China, but develops in a high speed. At present, some universities have gradually established the online virtual labs, and some have provided simulation services, such as online virtual laboratory in Mechanical Engineering College, Huazhong University of Science and Technology, Chemistry Laboratory, Wuhan Institute of Technology, and Modern Education Technology Virtual Laboratory in department of Education, Zhejiang Normal University. Guo J evaluated the advantages of online virtual experimental teaching and discussed the wide application of online virtual laboratory experiment as a new teaching method [5]. Huang $\mathrm{Y}$ et al reported a virtual laboratory of diagnostics with multimedia programming and virtual reality technology, which implements human-computer interaction experiments with innovation, interaction, intelligence, simulation, openness and scalability.

It has achieved remarkable results in enhancing experimental teaching results, training clinical techniques and clinical thinking for medical students, graduate students and young physicians [7]. Lin $G$ et al established an interactive technology platform by Flash, in combination with video, images and animation to create a virtual human-computer interaction experimental physiological system [8]. Zhang T et al used Flash to build a virtual laboratory of regional anatomy [9]. Sun D constructed virtual laboratory of histology and embryology [10]. Roignot $\mathrm{P}$ et al reported virtual slice technique was used as routine training in pathology laboratory [11]. In recent years, virtual reality technology has gradually been applied in all areas of university teaching, but the virtual laboratory of pathogen microbiology has not been reported.

In modern education, experiment is an important part of teaching. Traditional laboratory generally requires a lot of equipments. With the development of science and technology, equipment is upgraded in an increasingly rapid speed, and as the expansion of enrollment, the laboratory equipment purchase and update have become an important issue in experimental teaching reform. Medical microbiology is a very practical subject, and the "pathogenic micro-organisms laboratory bio-safety regulation" is demanding for medical microbiology laboratory that it is hard for many schools to achieve. The emergence and development of virtual reality technology provides a new way to solve the above problem. Virtual reality technology can establish a virtual laboratory, supplement or replace some traditional experiments that are too difficult to be carried out in traditional conditions. In addition, the use of
Internet technology helps both students and teachers operate in virtual laboratory at any time, which not only greatly improves the resource utilization, but also can be used in remote experimental teaching.

According to the State Council "pathogenic microorganisms laboratory bio-safety regulation", pathogen involved in the medical microbiology experiments belongs to the type III pathogen that infects human and needs to be handled in laboratory with at least type II biosafety protection. In this paper, two virtual experiments are established with fun, cartoon-style, combined with software, videos, pictures and animations, as well as human-computer interaction. The obstacles are set up as game operation to stimulate students' interests in learning. The database of software can be used to score automatically, and can keep the operator's information and feedback. The software is user-friendly, and can be used by many students simultaneously. After school, by playing in virtual experiment scenarios in a relaxed way, students can review experimental procedures and know how well they have learnt through score feedbacks. It can also be used for teaching evaluation and experimental assessment, which lays a foundation for the further construction of virtual laboratory of medical microbiology, with a wide range of applications.

\section{References}

[1] Hung Y, Vetivelu A, Hird M A, et al. Using fMRI virtual-reality technology to predict driving ability after brain damage: a preliminary report.Neurosci Lett, vol.13, no.558, pp41-46, 2014

[2] Chen F, Griffith A, Cottrell A, et al. Evaluation of virtual microscopy in medical histology teaching. Anat Sci Educ, vol.6, no.5,pp307-15,2013

[3] Wong Y L. Behavioral responses to epidemics in an online experiment: using virtual diseases to study human behavior. PLoS One.vol.8, no.1, pp.52814, 2013

[4] Yang Y. Building and application of Virtual laboratory, wireless internet technology, no.9, pp.50. 2013

[5] Guo J. New methods of experimental teaching-online virtual labs. Technological information, no.10, pp.143. 2006

[6] Game Based Learning in Universities and Lifelong Learning[EB/OL]. Available from:http:// www.Unigame.net/html/OnlineEduca_web.pdf. January 2005

[7] Huang Y, Wang Z, Xu W, et al., Construction and application of a virtual diagnosis laboratory. Northwest Medical Education, vol.15, no.5, pp.955-957, 2007

[8] Lin G, Xiang Q, Wang S, et al. Virtual experiments and its applications in physiology medical education, Explore of medical education, vol.6, no.10, pp.948-955, 2007

[9] Zhang T, Chen L and Chen X. Application of flash technology in human anatomy virtual lab. Chinese equipment. China, no.6, pp.13-14,2007

[10] Sun D, Xu J. Application of virtual Lab in histology and embryology experimental teaching. Modern educational technology, vol.31, no.21, pp. 47,2013

[11] Roignot P, Donzel J P and Brunaud M D. The use of virtual slides in the daily practice of a pathology laboratory, Ann Pathol, vol.31, no.2, pp.73-77, 2011 